\title{
Análisis de la huella de carbono en vías de pavimento flexible (asfalto): Vías de segundo orden en la provincia del Azuay
}

\author{
Analysis of the carbon footprint in flexible pavement roads (asphalt): \\ second order roads in the province of Azuay
}

Pablo Esteban Beltrán Castro. ${ }^{1}$, Karla Belén García Molina. ${ }^{2}$ \& Carlos Julio Cordero Cabrera. $^{3}$

\begin{abstract}
DOI: https://doi.org/10.33262/concienciadigital.v4i4.1.1927

Objective. Determine the major pollutants generated during the construction and maintenance stage of the Minas Tablón Pucará highway project. Methodology. $1 \mathrm{~km}$ of track was analyzed and all the factors generated by the activities during all phases of the project were established, using the SimaPro 9.1.1.1 software and the ReCiPe Midpoint methodology, different phases were distinguished as definition of the approach and limits of the system, inventory, impact analysis and interpretation of the results, with the guidelines of the ISO 14040 and 14044 standards, the environmental impacts were identified and evaluated during all stages of the life cycle. In addition, through the

\footnotetext{
1 Universidad Católica de Cuenca, Maestría en Construcciones con Mención en la Administración de Construcción Sustentable, Azuay, Ecuador, pablo.beltran1@est.ucacue.edu.ec, ORCID https://orcid.org/0000-0001-5040-3965.

2 Universidad Del Azuay, Maestría en Gestión Ambiental. Cuenca, Ecuador, karlagarcia@es.uazuay.edu.ec. ORCID https://orcid.org/0000-0002-5270-8890.

3 Universidad Católica de Cuenca, Maestría en Construcciones con Mención en la Administración de Construcción Sustentable, Azuay, Ecuador, carlos.cordero@ucacue.edu.ec, ORCID https://orcid.org/0000-0002-6373-6280.
} 
geographic information system ARC-GIS, the shapes of the Ministerium Ministry of Agriculture and Livestock 25k, 2018 and the georeferenced points taken in the field, the coverage and land use corresponding to the area where the project is located was determined. Results. Among the results with the highest contamination is the maintenance stage, which generates a high rate of climate change $(67.1 \%)$, fossil depletion (68\%), particulate matter (94.1\%) and human toxicity $(90.2 \%)$. Conclusion. During the maintenance stage, human interventions are more periodic so more contamination is generated, in addition, the study route is asphalt so it presents more chemicals and compounds, even with regard to the construction methodology generates an increase in emissions that contribute considerably to global warming and climate change.

Keywords: carbon footprint, road, pavement, construction, maintenance, emissions.

\section{Resumen}

Objetivo. Determinar los mayores contaminantes generados durante la etapa de construcción y mantenimiento del proyecto vial Minas- Tablón - Pucará. Metodología. Se analizó $1 \mathrm{Km}$ de vía y se estableció todos los factores que se generan por las actividades durante todas las fases del proyecto, mediante el software SimaPro 9.1.1.1 y la metodología de ReCiPe Midpoint se distinguió diferentes fases como definición del enfoque y límites del sistema, inventario, análisis del impacto y la interpretación de los resultados, con lineamientos de las normas ISO 14040 y 14044, se identificó y evaluó los impactos ambientales durante todas las etapas del ciclo de vida. Además, mediante el sistema de información geográfica ARC-GIS, los shape del Ministerio de Agricultura y Ganadería MAG 25k 2018 y los puntos de georreferencia tomados en campo se determinó la cobertura y uso de suelo que corresponde a la zona donde se ubica el proyecto. Resultados. Entre los resultados que mayor contaminación presentan se encuentra la etapa de mantenimiento, esta genera un alto índice de cambio climático $(67.1 \%)$, agotamiento fósil (68\%), material particulado (94.1\%) y toxicidad humana $(90.2 \%)$. Conclusión. Durante la etapa de mantenimiento las intervenciones humanas son más periódicas por lo que se generan mayor contaminación, además, la vía de estudio es de asfalto por lo que se presenta mayores químicos y compuestos, incluso a lo que refiere a la metodología constructiva genera incremento de emisiones que aporta considerablemente al calentamiento global y cambio climático.

Palabras claves: huella de carbono, vía, pavimento, construcción, mantenimiento, emisiones.

\section{Introducción}

El calentamiento global en los últimos años ha tenido una evolución que afecta principalmente a la temperatura de la atmósfera y a los océanos, el hielo de los glaciares y nevados continúan derritiéndose y el nivel del mar sigue en incremento (Andrade \& 
Segura, 2014). El incremento de la concentración de gases que generan el efecto invernadero tales como el dióxido de carbono, ozono, metano y óxido nitroso, han sido uno de los factores principales que incrementa esta problemática global (Trenberth, 2007). Este tipo de gases impiden que las radiaciones ultravioletas se redirijan hacia el espacio y se queden retenidas en la tierra provocando el incremento de la temperatura (Espíndola \& Valderrama, 2012b).

Dentro de los factores involucrados en el incremento de la temperatura ambiental se encuentran: variación de los niveles del mar, incremento de las concentraciones del dióxido de carbono, vapor de agua, metano y sus hidratos, variación de la reflexión y el campo magnético de la tierra, así como la cantidad de energía solar (Climático, 2007). Las principales industrias que generan estos cambios son los que trabajan con energía, medios de transporte y de producción masiva (Díaz, 2012).

La huella de carbono (HdC), es una medida que estima la cantidad de los gases que provocan el efecto invernadero que se emiten a la capa atmosférica generados principalmente por las actividades del ser humano (Espíndola \& Valderrama, 2012a). Esta expresada en unidades equivalentes de $\mathrm{CO}_{2}$, está conformado por cada una de las actividades para la realización de un producto, tomando en cuenta las materias primas y los residuos generados (Vigizzo, 2010). Para la determinación de la huella de carbono es importante determinar las principales fuentes generadoras de los gases de efecto invernadero (GEI). En la tabla 1, se evidencia las emisiones de gases de efecto invernadero por el Ecuador. Este es un valor que conforme pasan los años va en aumento debido principalmente al incremento poblacional y a la evolución industrial.

Tabla 1

\begin{tabular}{cc} 
Emisiones de gases de efecto invernadero en el Ecuador \\
\cline { 2 - 2 } Año & $\begin{array}{c}\text { Concentraciones de } \\
\text { dióxido de carbono } \\
\mu \mathrm{g} / \mathrm{m} 3\end{array}$ \\
\hline 2008 & 47603,31 \\
2009 & 49448,33 \\
2010 & 50320,69 \\
2011 & 51593,66 \\
2012 & 52746,57 \\
\hline
\end{tabular}

Fuente: Carrasco (2017)

La construcción de vías conlleva a un desarrollo general de las comunidades, sin embargo, también presentan problemas ambientales debido al elevado consumo de materia prima, la utilización de combustibles fósiles y el cambio de uso de suelo. Al construir vías de asfalto los elementos ambientales que sufren mayor impacto adverso significativo son el agua, el aire y el suelo, por lo que en la actualidad existen organismo encargados de velar por el bienestar ambiental, aplicando la normativa ambiental ecuatoriana vigente referente a la emisión de gases de efecto invernadero y sugiriendo metodologías constructivas sustentables. 
En los pavimentos de asfalto el material de cementación es el cemento asfáltico de petróleo (CAP) o llamado Bitumen, tiene alto contenido de carbono que en promedio es del orden de $82 \%$, y el CAP constituye $5 \%$ de un pavimento de asfalto, siendo el resto los agregados - piedra, arena y grava (Bisso, 2018).

Existen varias normativas a nivel nacional que rigen el tema ambiental:

La Constitución de la República del Ecuador defiende la concepción de un ambiente sano siendo considerado como un derecho para la ciudadanía por medio de la cal se debe garantizar una vida sana con equilibrio ecológico y en base al buen vivir (Constitutivos \& Estado, 2018).

La ley de prevención y control de la contaminación de un ecosistema controla el desarrollo industrial y mantiene un equilibrio entre el desarrollo tecnológico y los recursos ambientales. Por medio de esta ley se estableció que se prohíbe las descargas directas al aire, agua y suelo de toda sustancia que sea contaminante y que ponga en riesgo la salud de los humanos (Lexis, 2008).

La ley de gestión ambiental contiene todos los puntos necesarios para que la producción inicie sus actividades y se mantengan dentro de los parámetros exigidos por el estado para que se conserven el medio ambiente por medio del desarrollo sustentable (Ambiental et al., 2004).

En el Código Orgánico Ambiental, articulo 2 se establece que se deben regular las actividades que generen impacto y daño ambiental, a través de normas y parámetros que promuevan el respeto a la naturaleza, a la diversidad cultural, así como a los derechos de las

generaciones presentes y futuras (De et al., 2017).

La norma técnica ecuatoriana para las emisiones al aire fue escrita bajo la Ley de Gestión Ambiental y del Reglamento a la Ley de Gestión Ambiental para la prevención y control de la Contaminación Ambiental. A continuación, se detallan los límites máximos permisibles de emisiones hacia el aire a partir de una fuente fija de combustión $\left(\mathrm{mg} / \mathrm{Nm}^{3}\right)$ bajo condiciones normales: 1013 milibares de presión, $0^{\circ} \mathrm{C}$ de temperatura, a un $7 \%$ de oxígeno para fuentes fijas cerradas y un $18 \%$ cuando son fuentes fijas abiertas (Registro oficial $\mathrm{N}^{\circ} 387,2015$ ).

Tabla 2

Valores Máximos permisibles de emisiones al Aire para Fuentes fijas de combustión

\begin{tabular}{cccc}
\hline $\begin{array}{c}\text { Contaminante } \\
\text { emitido }\end{array}$ & $\begin{array}{c}\text { Tipo de } \\
\text { combustible } \\
\text { utilizado }\end{array}$ & Unidades & $\begin{array}{c}\text { Valores máximos } \\
\text { permisibles de } \\
\text { emisiones de aire }\end{array}$ \\
\hline Material & Sólido & $\mathrm{mg} / \mathrm{Nm}^{3}$ & 150 \\
particulado & Búnker & $\mathrm{mg} / \mathrm{Nm}^{3}$ & 150 \\
& Diésel & $\mathrm{mg} / \mathrm{Nm}^{3}$ & 100 \\
\hline
\end{tabular}




\section{Tabla 2}

Valores Máximos permisibles de emisiones al Aire para Fuentes fijas de combustión (continuación)

\begin{tabular}{cccc}
\hline $\begin{array}{c}\text { Contaminante } \\
\text { emitido }\end{array}$ & $\begin{array}{c}\text { Tipo de } \\
\text { combustible } \\
\text { utilizado }\end{array}$ & Unidades & $\begin{array}{c}\text { Valores máximos } \\
\text { permisibles de } \\
\text { emisiones de aire }\end{array}$ \\
\hline Sólido & $\mathrm{mg} / \mathrm{Nm}^{3}$ & 850 \\
Nitrógeno & Búnker & $\mathrm{mg} / \mathrm{Nm}^{3}$ & 550 \\
& Diésel & $\mathrm{mg} / \mathrm{Nm}^{3}$ & 500 \\
Dióxido de azufre & Búnker & $\mathrm{mg} / \mathrm{Nm}^{3}$ & 140 \\
& Diésel & $\mathrm{mg} / \mathrm{Nm}^{3}$ & 1650 \\
& Sólido & $\mathrm{mg} / \mathrm{Nm}^{3}$ & 1650 \\
& Búnker & $\mathrm{mg} / \mathrm{Nm}^{3}$ & 1800 \\
Monóxido de & Diésel & $\mathrm{mg} / \mathrm{Nm}^{3}$ & 300 \\
carbono & Gaseoso & $\mathrm{mg} / \mathrm{Nm}^{3}$ & 200 \\
& & & 100 \\
\hline
\end{tabular}

Fuente: Registro oficial N³87 (2015), Tomado de: Texto Unificado de Legislación Secundaria del Ministerios del Ambiental

\section{Metodología}

La metodología tiene un tipo de diseño preexperimental donde el nivel de estudio del caso es a través de medición; se trata del análisis del ciclo de vida y se encuentra descrita en las normas ISO 14040 y 14044.

Para este análisis también se consideró el uso y cobertura de suelo que existe en la zona, los cuerpos de agua, la flora y fauna, toda esta información obtenida a través de los shape del Ministerios de Agricultura y Ganadería MAG 25k 2018, y SENAGUA.

El inventario del ciclo de vida es la recolección de toda la información de tipo numérico en base al ciclo de vida del sistema, es decir, emisiones de $\mathrm{CO} 2$ para la determinación de a huella de carbono.

El Proyecto vial Minas - Tablón Pucará se encuentra en la provincia del Azuay, cantón Pucará y Santa Isabel, que tiene una longitud de intervención de 36 km, sin embargo, la unidad funcional que se consideró es 1 kilómetro de la carretera asfaltada durante un mes de operación. 


\section{Figura 1}

Ubicación del proyecto vial Minas-Tablón-Pucará 36Km
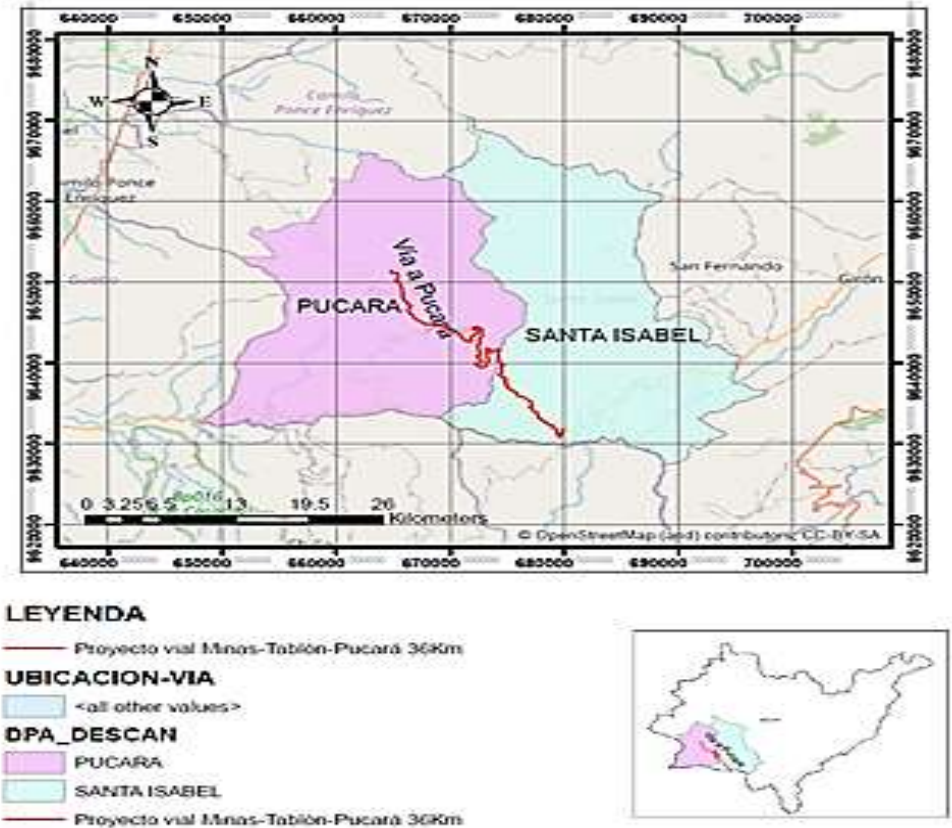

Nota: Proyecto vial Minas - Tablón - Pucará 36km

\section{Hidrografía}

Se encuentra constituida por microcuencas, ríos y quebradas; la mayoría escasos en verano y torrentosos en invierno, muy importantes para la población local, puesto que son fuentes de captación de agua para consumo doméstico, riego y abrevaderos de animales.

Estas características se detallan a continuación en la siguiente figura:

\section{Figura 2}

Ríos y quebradas que intervienen en el proyecto vial Minas-Tablón-Pucará 36Km
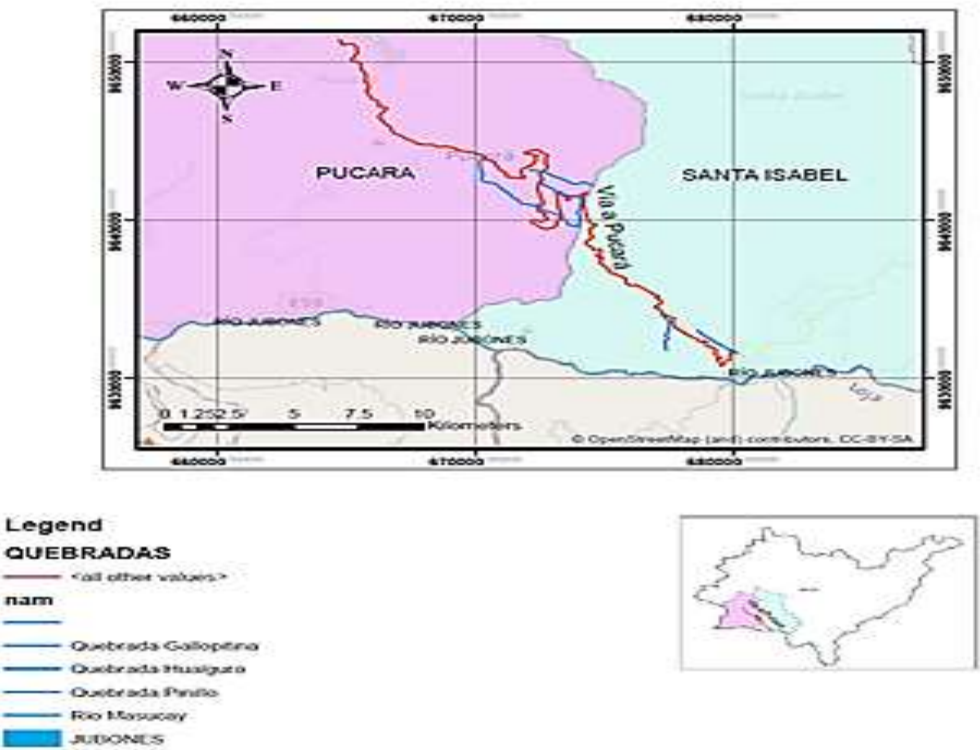

Nota: Ríos y Quebradas que intervienen en el proyecto vial Minas - Tablón - Pucará $36 \mathrm{~km}$ 


\section{Vegetación}

La vegetación corresponde a Matorral seco montano (valles secos, árboles dispersos, tierra apta para agricultura) y Matorral húmedo montano (lugares húmedos, se encuentra en el Callejón interandino, vegetación original en su mayoría destruida y reemplazada por cultivos y bosques de Eucaliptus globulus.

En su mayor parte atraviesa el Bosque de vegetación protectora BVP, y parte del páramo del Azuay.

\section{Figura 3}

Ecosistemas del Proyecto Vial Minas-Tablón-Pucará 36Km
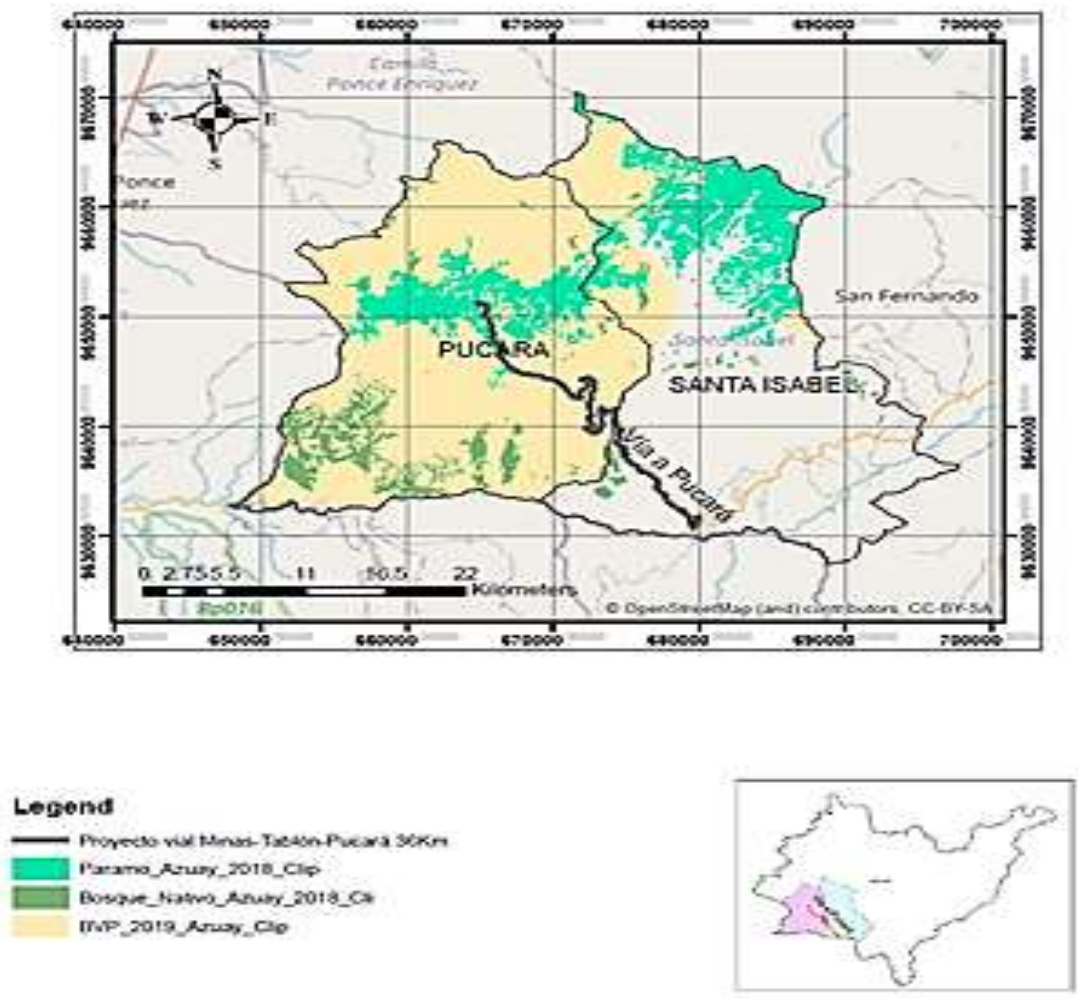

Nota: Ecosistemas del proyecto vial Minas - Tablón - Pucará 36km Uso y Cobertura del suelo del Suelo

El uso del suelo ha sido transformado de bosques a extensos campos agrícolas, ganaderos, pastos y potreros.

En el mapa se puede apreciar que gran parte de la zona es de pastizal. 


\section{Figura 4}

Uso y Cobertura del Suelo del Proyecto vial Minas-Tablón-Pucará 36 Km
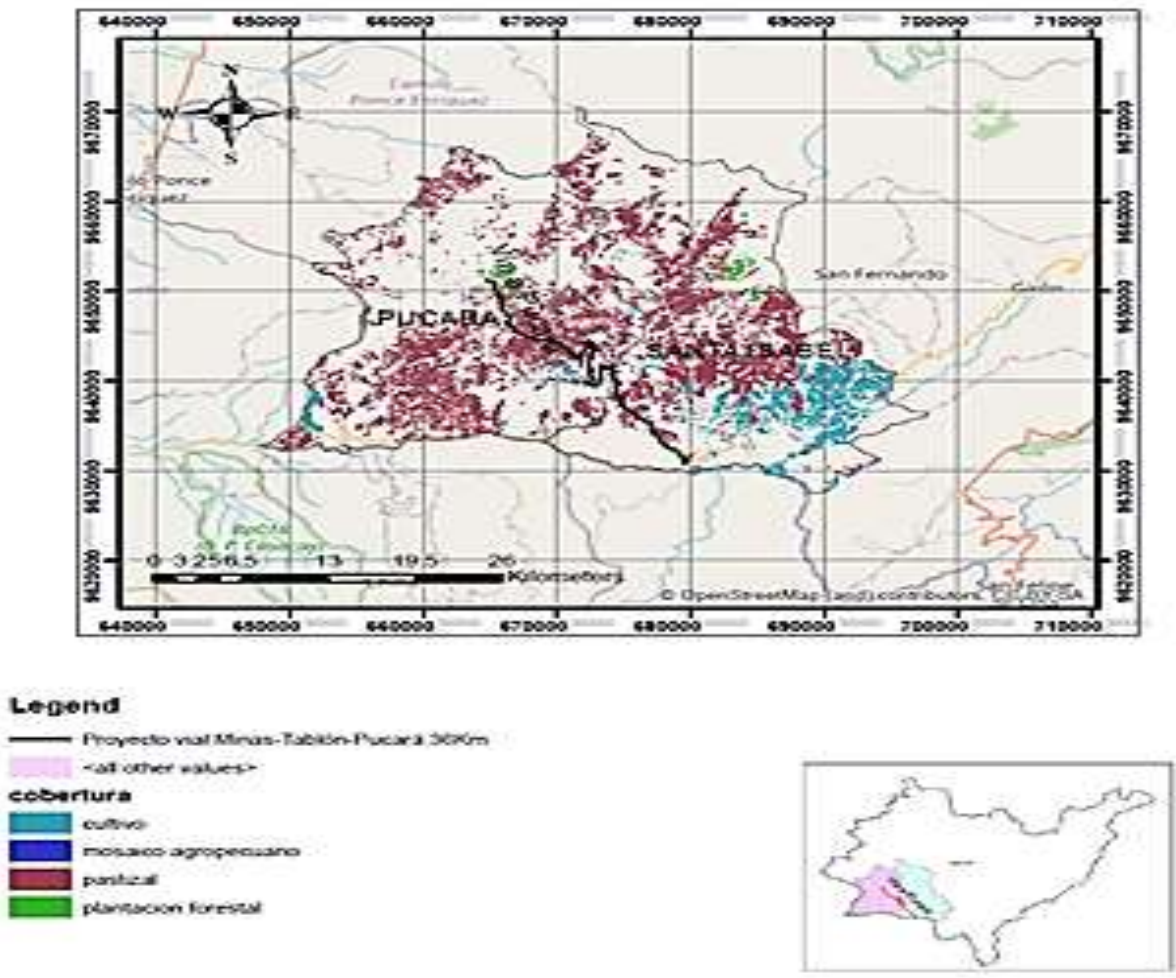

Nota: Uso y cobertura del suelo del proyecto vial Minas - Tablón - Pucará 36km

\section{Actividades en la fase de Construcción}

1. Movimiento de tierras y limpieza lateral de vegetación

2. Establecimiento y funcionamiento de campamento para personal de la obra

3. Construcción y/o adecuación de patio para mantenimiento de maquinaria

4. Adecuación de áreas para funcionamiento de la trituradora y planta de asfaltos

5. Construcción de obras complementarias a la vía: cunetas, muros, puentes y otros

6. Construcción de calzada: Colocación de sub-base, base y carpeta asfáltica

7. Movimiento de vehículos y maquinarias

8. Transporte de materiales pétreos y áridos

\section{Actividades en la fase de mantenimiento}

1. Construcción y/o adecuación de patio para mantenimiento de maquinaria

2. Adecuación de áreas para funcionamiento de la trituradora y planta de asfaltos

3. Mantenimiento de obras complementarias a la vía: cunetas, muros, puentes y otros

4. Mantenimiento a nivel carpeta asfáltica

5. Movimiento de vehículos y maquinarias

6. Transporte de materiales pétreos y áridos

\section{Pasos definidos en el software}

1.- Se definió el objetivo y el alcance 
2.- Se escogió las bibliotecas para el análisis del caso de estudio (Ecoinvent 3, ELCD y EU\&DK Input Output Database y Industry data2 y Methods)

3.- Se estableció entradas y salidas en el sistema y el inventario

4.- Se evaluó el impacto, se obtuvo los resultados y se interpretó.

\section{Figura 5}

Selección de entradas y Salidas

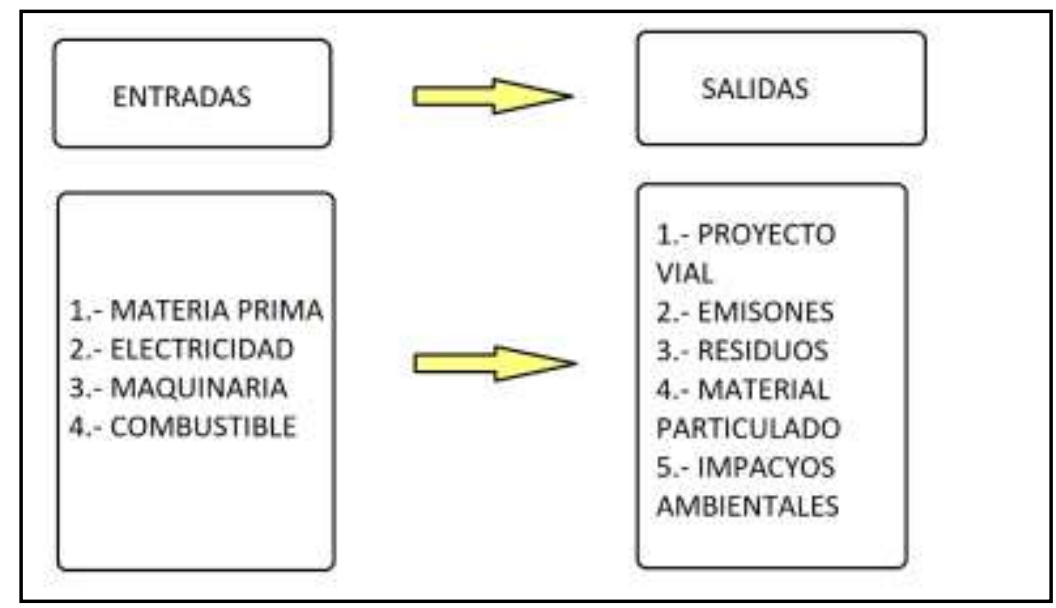

Nota: Metodología del programa SimaPro para el proyecto vial Minas - Tablón - Pucará 36km

\section{Resultados}

Luego de haber identificado las variables dependientes de la zona de estudio y el tipo de proyecto, se introdujo todos los datos y parámetros necesarios para determinar el ciclo de vida el software SimaPro y se obtuvo la siguiente tabla de resultados la cual detalla el impacto ambiental generado en sus 2 etapas, construcción y mantenimiento, como se muestra en la tabla 3.

\section{Tabla 3}

Impactos ambientales por categoría de impacto. Resultado referido a la unidad funcional: $1 \mathrm{Km}$ de carretera

\begin{tabular}{|c|c|c|c|c|}
\hline \multirow{2}{*}{ IMPACTOS AMBIENTALES } & \multirow{2}{*}{ UNIDAD } & \multicolumn{2}{|c|}{ ETAPA } & \multirow{2}{*}{ TOTAL } \\
\hline & & CONSTRUCCION & MANTENIMIENTO & \\
\hline ACIDIFICACIÓN TERRESTRE & $\mathrm{kg} \mathrm{SO} 2 \mathrm{eq}$ & 5.60 & 12.54 & 18.14 \\
\hline AGOTAMIENTO DE AGUA & $\mathrm{m} 3$ & 4.10 & 5.88 & 9.98 \\
\hline AGOTAMIENTO DE METALES & $\mathrm{kg} \mathrm{Fe}$ eq & 30.27 & 356.12 & 386.39 \\
\hline AGOTAMIENTO DE OZONO & mg CFC-11 eq & 132.54 & 355.70 & 488.24 \\
\hline AGOTAMINETO FÓSIL & $\mathrm{kg}$ oil eq & 318.10 & 674.45 & 992.55 \\
\hline CAMBIO CLIMÁTICO & $\mathrm{kg} \mathrm{CO} 2 \mathrm{eq}$ & 982.13 & 2006.74 & 2988.87 \\
\hline ECOTOXICIDAD DE AGUA DULCE & $\mathrm{kg} 1,4-\mathrm{DB}$ eq & 3.10 & 23.43 & 26.53 \\
\hline ECOTOXICIDAD TERRESTRE & $\mathrm{kg} 1,4-\mathrm{DB}$ eq & 0.52 & 1.56 & 2.08 \\
\hline EUTROFIZACIÓN DE AGUA DULCE & g P eq & 51.67 & 378.2 & 429.87 \\
\hline FORMACION DE OXIDANTES FOTOQUÍMICOS & kg NMVOC & 4.79 & 11.42 & 16.21 \\
\hline
\end{tabular}




\section{Tabla 3}

Impactos ambientales por categoría de impacto. Resultado referido a la unidad funcional: $1 \mathrm{Km}$ de carretera (continuación)

\begin{tabular}{|c|c|c|c|c|}
\hline \multirow{2}{*}{ IMPACTOS AMBIENTALES } & \multirow{2}{*}{ UNIDAD } & \multicolumn{2}{|c|}{ ETAPA } & \multirow{2}{*}{ TOTAL } \\
\hline & & CONSTRUCCION & MANTENIMIENTO & \\
\hline MATERIAL PARTICULADO & kg MP10 eq & 50.64 & 802.45 & 853.09 \\
\hline OCUPACION DE SUELO AGRICOLA & ha*a & 3.87E-03 & $9.89 \mathrm{E}-03$ & 0.01376 \\
\hline OCUPACION DE SUELO URBANO & ha*a & 5.92E-04 & $1.94 \mathrm{E}-03$ & 0.002532 \\
\hline RADIACIÓN IONIZANTE & $\mathrm{kBq} \mathrm{U} 235 \mathrm{eq}$ & 58.00 & 153.59 & 211.59 \\
\hline TRASNFORMACION DE TERRENO NATURAL & ha & $3.11 \mathrm{E}-05$ & $8.23 \mathrm{E}-05$ & 0.0001134 \\
\hline TOXICIDAD HUMANA & kg 1,4-DB eq & 74.6 & 683.51 & 758.11 \\
\hline
\end{tabular}

En la tabla 3 podemos observar como la intervención de una obra civil, que en este caso es la construcción y mantenimiento de una vía puede generar acumulación de gases de efecto Invernadero, mismos que producen el calentamiento global.

Dependiendo del tipo de material que se utilice, la metodología constructiva y maquinaria empleada se puede aumentar o disminuir estos problemas de huella ecológica.

Entre los impactos más significativos en todas las etapas de intervención se tiene:

\section{Figura 6}

\section{Mayores Impactos Ambientales}

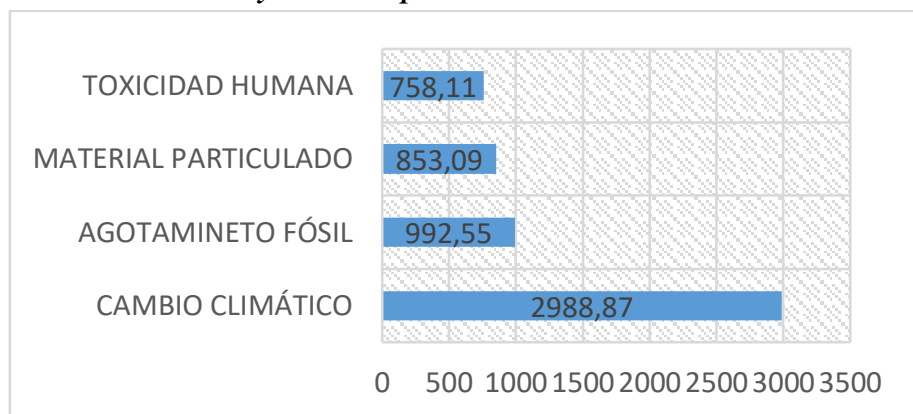

En la figura 6 se detalla los mayores impactos ambientales que se producen en 1 kilómetro del Proyecto Vial Minas-Tablón-Pucará. El mayor impacto producido es el cambio climático con un valor de 2988,87 kilogramos, el agotamiento fósil se encuentra en segundo lugar con un valor igual a 992,55kilogramos, seguido del material particulado con un valor de 853,09 kilogramos y finalmente se encuentra la toxicidad humana con un valor de 758,11 kilogramos.

Debido a las sustancias químicas que se utilizan para fabricar el asfalto y las emisiones que se emiten al momento de vaciar en obra, producen toxicidad humana mismos que conllevan a grandes problemas de salud tanto al personal que se encuentra laborando en el proyecto, como a las comunidades aledañas a la zona de intervención. Así como también el material particulado que genera el equipo caminero al momento de la ejecución del proyecto, es uno de los principales contaminantes del aire que ocasionan dificultades respiratorias a las personas y animales. 
El agotamiento fósil que se genera debido a las actividades de intervención y maquinaria utilizada, producen un alto impacto ambiental, la quema de estos combustibles emiten dióxido de carbono produciendo un incremento considerable en el calentamiento global.

Todos estos gases de efecto invernadero que se generan durante la intervención humana producen el cambio climático, en la tabla podemos observar el alto grado de impacto que se genera, ocasionando alteraciones a los parámetros climáticos.

Además, se observa que la mayor parte de impactos ambientales se encuentran vinculados con la etapa de mantenimiento vial, como se observa en la figura 7.

\section{Figura 7}

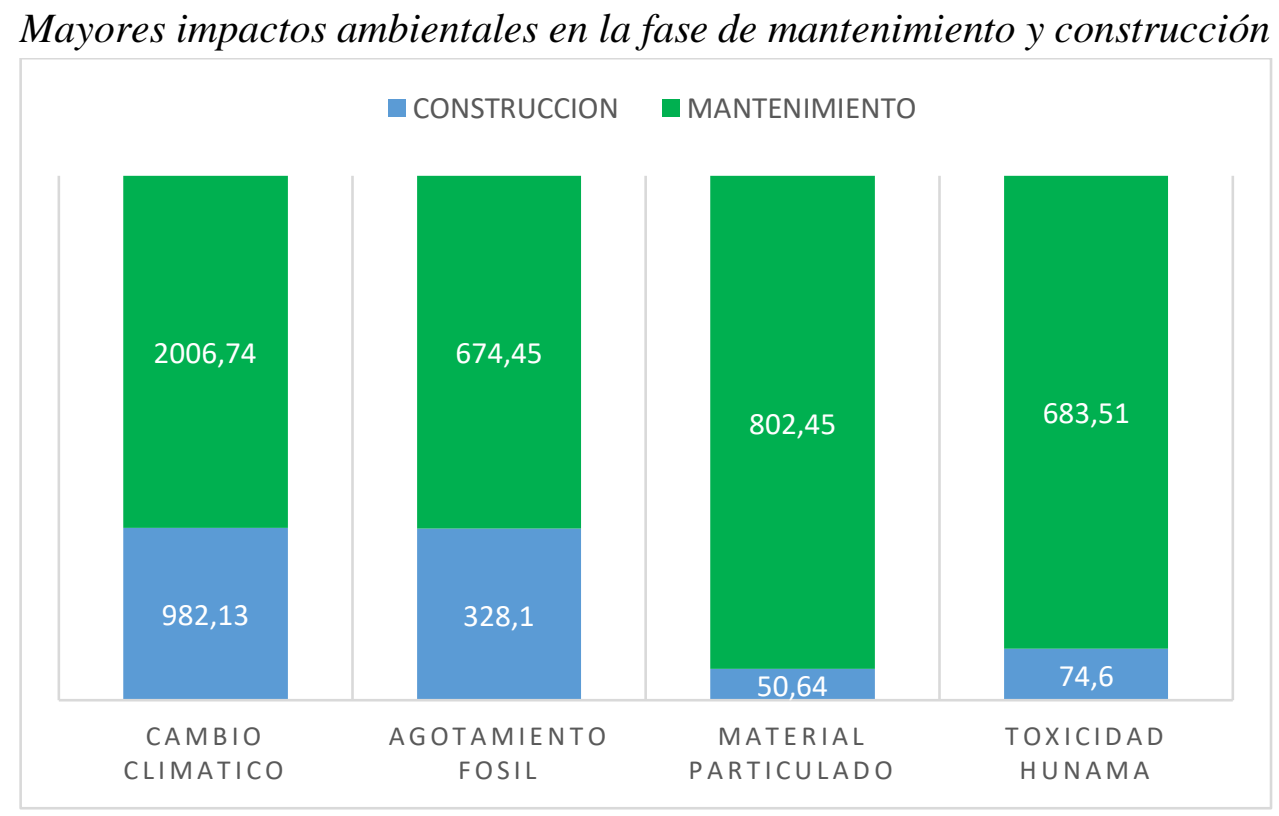

En todos los casos podemos observar que el mayor impacto ambiental se genera en la fase de mantenimiento, debido al tipo de clima, suelo y uso de carretera exige que el mantenimiento tenga periodicidad anual, esto ocasionando que la contaminación sea frecuente.

Con estos resultados se puede verificar que el mayor impacto ambiental corresponde al cambio climático, debido a las emisiones tóxicas generadas por las distintas actividades humanas, a continuación, se encuentra el agotamiento fósil debido al consumo y uso excesivo de los recursos para la producción del asfalto y la actividad en sí. Luego se encuentra el material particulado generado por los vehículos, maquinaria y las actividades de producción y colocación. Finalmente está la toxicidad humana que depende de diferentes factores: dosis, duración y ruta de exposición afectando tanto a los trabajadores durante el proceso de construcción y mantenimiento. Sin embargo, existen otros problemas ambientales que se generan en menor grado, pero con igual riesgo de contaminación. Así mismo se observó claramente que durante el mantenimiento es cuando se da mayor contaminación. 


\section{Conclusiones}

- Como se puede apreciar en los resultados obtenidos, la intervención humana para realizar proyectos en general producen impactos ambientales a diferentes escalas, dependiendo de la zona a intervenir, si es una área de bosque producirá más afección que una zona industrial, también se debe considerar los materiales que se utilizarán ya que cada uno tiene diferentes compuestos químicos que generan mayor riesgos en la salud que otros; un factor muy importante a considerar es el equipo y maquinaria a utilizar, mientras más antiguo sea la maquinaria y menos mantenimientos se hayan dado, estas generaran mayor contaminación al aire, suelo y agua, como se observa en la tabla 4.

\section{Tabla 4}

Comparación de resultados durante las fases estudiadas

\begin{tabular}{ccccccccc}
\hline & \multicolumn{2}{c}{$\begin{array}{c}\text { CAMBIO } \\
\text { CLIMÁTICO }\end{array}$} & \multicolumn{2}{c}{$\begin{array}{c}\text { AGOTAMIENTO } \\
\text { FÓSIL }\end{array}$} & \multicolumn{2}{c}{$\begin{array}{c}\text { MATERIAL } \\
\text { PARTICULADO }\end{array}$} & \multicolumn{2}{c}{$\begin{array}{c}\text { TOXICIDAD } \\
\text { HUMANA }\end{array}$} \\
\hline construcción & 982.13 & $32.9 \%$ & 318.1 & $32.0 \%$ & 50.64 & $5.9 \%$ & 74.6 & $9.8 \%$ \\
mantenimiento & 2006.74 & $67.1 \%$ & 674.45 & $68.0 \%$ & 802.45 & $94.1 \%$ & 683.51 & $90.2 \%$ \\
total & 2988.87 & $100 \%$ & 992.55 & $100 \%$ & 853.09 & $100 \%$ & 758.11 & $100 \%$ \\
\hline
\end{tabular}

- Con la investigación realizada en este proyecto se pudo concluir que la etapa de mantenimiento genera mayor impacto ambiental, tanto así que el cambio climático en dicha fase representa un $67.1 \%$ de contaminación y un 32,9\% en la etapa de construcción, esto debido a que para conservar la estructura de calzada se planifica intervenciones de manera periódica, ocasionando gases de efecto invernadero que incrementan la temperatura de la tierra. Dentro de la fase de mantenimiento el agotamiento fósil representa el $68 \%$ comparado al de construcción con $32 \%$, debido a que cada vez se necesita la maquinaria y recursos naturales para poder intervenir, además durante las ejecuciones se genera material particulado PM10 y PM2.5 pero durante el mantenimiento este contaminante es mayor que en construcción, como podemos observar, con el $94.1 \%$ y $5.9 \%$ respectivamente. Así mismo en lo que refiere a la toxicidad humana tenemos el $90.2 \%$ de contaminación en la fase de mantenimiento y el $9.8 \%$ durante la construcción.

- Es por ello por lo que se debe generar concientización a todo el personal que va a laborar dentro de un proyecto, es responsabilidad de todos generar buenas prácticas ambientales de manera que aportemos a la humanidad a disminuir los gases de efecto invernadero que están elevando al indicador ambiental de la huella de carbono.

- Los involucrados internos y externos de un proyecto deben generar propuestas para lograr un desarrollo sostenible organizacional, considerando la normativa técnica ecuatoriana para las emisiones al aire a partir de una fuente fija de combustión escrita bajo la Ley de Gestión Ambiental y del Reglamento a la Ley de Gestión Ambiental para la prevención y control de la Contaminación Ambiental. 
- En la tabla 2, se detallan los límites máximos permisibles de emisiones hacia el aire a partir de una fuente fija de combustión, las unidades de medición son $\mathrm{mg} / \mathrm{Nm}^{3}$ que significa miligramos por metro cúbico de gas bajo condiciones normales: 1013 milibares de presión, $0^{\circ} \mathrm{C}$ de temperatura, en base seca, a un $7 \%$ de oxígeno para fuentes fijas cerradas y un $18 \%$ cuando son fuentes fijas abiertas.

- La rama de la ingeniería civil está altamente ligada al tema ambiental, la ejecución de proyectos viales son uno de los principales responsables del calentamiento global, es por ello por lo que debe ir de la mano las metodologías constructivas con los planes de manejo ambiental, de esta manera generar construcciones sostenibles y sustentables que impliquen uso eficiente de los recursos naturales disminuyendo la degradación ambiental y asegurando una vida digna y saludable a las generaciones futuras.

\section{Agradecimientos}

El presente artículo es parte del trabajo de investigación y titulación del Programa de Maestría en Construcción con Mención en Administración de la Construcción Sustentable de la Universidad Católica de Cuenca, por ello agradecemos a los profesores, instructores y cada una de las personas que intervinieron en el proceso de maestría, también cabe agradecer al Ministerio de Transporte y Obras Publicas por la información y ayuda proporcionada.

\section{Referencias Bibliográficas}

Ambiental, L. E. Y. D. E. G., Comision, L. A., Codificacion, D. E. L. Y., Principios, A. Y., \& Gestion, D. E. L. A. (2004). Ley de gestion ambiental, codificacion. 1-14.

Andrade, H. J., Campo, O., \& Segura, M. (2014). Carbon footprint of the rice (Oryza sativa) production system in the municipality of Campoalegre, Huila, Colombia CAMBIO CLIMÁTICO RESUMEN. In Corpoica Cienc. Tecnol. Agropecu (Vol. 15, Issue 1). Corporacion Colombiana de Investigación Agropecuaria. https://dialnet.unirioja.es/servlet/articulo?codigo=5624645\&info=resumen\&idioma $=\mathrm{SPA}$

Bisso, R. (2018). Construcción de Pavimentos Sostenibles y la Huella de Carbono. Tecnología Limpia para obras viales, 55-89. Obtenido de http://liganteasfaltico.blogspot.com/2012/01/8-construccion-de-pavimentos.html

Carrasco, M. (2017). Plan de Manejo Ambiental para la Unidad Desconcentrada de Terminales de Ambato a fin de Mitigar la Huella de Carbono. Universidad Técnica de Ambato, Ambato. Obtenido de https://repositorio.uta.edu.ec/bitstream/123456789/25117/1/BQ\%20109.pdf

Climático, Y. E. L. C. (2007). Instituto de Hidrología, Meteorología y Estudios Ambientales - IDEAM SUBDIRECCIÓN DE METEOROLOGÍA 1.

Constitutivos, E., \& Estado, D. E. L. (2018). Constitucion de la republica del ecuador. 
$1-222$.

De, R. O. S., Del, H., \& Barrezueta, P. (2017). Codigo organico del ambiente. 1-92.

Díaz, G. (2012). El cambio climatico. Ciencia y Sociedad, 37, 227-240. http://repositoriobiblioteca.intec.edu.do/bitstream/handle/123456789/1392/CISO20 123702-227-240.pdf? sequence $=1 \&$ isAllowed $=y$

Espíndola, C., \& Valderrama, J. O. (2012a). Huella del carbono. Parte 1: conceptos, métodos de estimación y complejidades metodológicas. Informacion Tecnologica, 23(1), 163-176. https://doi.org/10.4067/S0718-07642012000100017

Espíndola, C., \& Valderrama, J. O. (2012b). Huella del carbono. Parte 2: La visión de las empresas, los cuestionamientos y el futuro. Informacion Tecnologica, 23(1), 177192. https://doi.org/10.4067/S0718-07642012000100018

Lexis. (2008). CONSTITUCION DE LA REPUBLICA DEL ECUADOR 2008 Decreto Legislativo 0 Registro Oficial. In Constitución del Ecuador (p. 136). www.lexis.com.ec

Registro oficial N³87. (2015). Norma De Emisiones Al Aire Desde Fuentes Fijas De Combustion Libro Vi Anexo 3. Texto Unificado de Legislación Secundaria Del Ministerio Del Ambiente, 097, 6-26.

Trenberth, K. E. (2007). Observations: surface and atmospheric climate change. Changes, 164(236-336), 235-336. https://doi.org/10.5194/cp-6-379-2010

Vigizzo, E. (2010). Huella de carbono, ambiente y agricultura en el cono sur de Suramérica.

ICCA.

Obtenido

de

https://books.google.es/books?hl=es\&lr=\&id=TIyUlyosD1sC\&oi=fnd\&pg=PA7 $\& d q=$ huella + de + carbono $\&$ ots $=V v 50$ Ejueb\&sig=hg-

DgnjVJFLKNnA3olskNmOb9gU\#v=onepage \&q=huella\%20de\%20carbono\&f= false

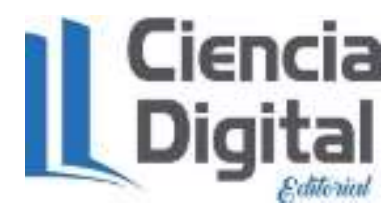




\section{PARA CITAR EL ARTÍCULO INDEXADO.}

Beltrán Castro, P. E., García Molina, K. B., \& Cordero Cabrera, C. J. (2021). Análisis de la huella de carbono en vías de pavimento flexible (asfalto): Vías de segundo orden en la provincia del Azuay. ConcienciaDigital, 4(4.1), 81-95. https://doi.org/10.33262/concienciadigital.v4i4.1.1927

\section{Liencia}

El artículo que se publica es de exclusiva responsabilidad de los autores y no necesariamente reflejan el pensamiento de la Revista Conciencia Digital.

El artículo queda en propiedad de la revista y, por tanto, su publicación parcial y/o total en otro medio tiene que ser autorizado por el director de la Revista Conciencia Digital.

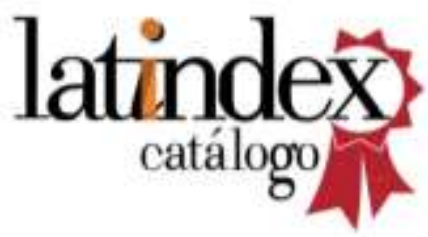

